\title{
Estetizar los cuerpos activados: teatro y militancia en torno al Cordobazo
}

\author{
Lorena Verzero \\ CONICET-Universidad de Buenos Aires, Argentina \\ lorenaverzero@gmail.com
}

\section{Resumen:}

El contexto cordobés en torno a 1969 fue especialmente fértil para la emergencia de prácticas artísticas militantes. En el marco de la Universidad Nacional de Córdoba movilizada por los acontecimientos sociales y políticos, surgió Libre Teatro Libre (LTL), que fue luego uno de los grupos de teatro militante más reconocidos de la época. Realizaré un recorrido crítico por el proceso político en torno al Cordobazo a través del análisis de la experiencia de LTL, poniendo de relieve aspectos significativos que operan como metonimia (por asociación o por contraste) de otras experiencias, para reflexionar en torno a problemáticas como las conexiones entre militancia y experimentación; las apuestas estéticas en función de los objetivos artístico-políticos; entre otras.

Palabras Clave: Cordobazo, Militancia teatral, Cuerpos, Estética, Política.

\section{Abstract:}

The Cordoba context around 1969 was especially fertile for the emergence of activist artistic practices. The group Libre Teatro Libre (LTL) emerged from the National University of Córdoba at the right moment when it was mobilized by social and political events. LTL was later one of the most recognized militant theater groups of the time. I will develop a critical approach to the political process around the Cordobazo through the analysis of the experience of LTL, highlighting significant aspects that operate as metonymy (by association or by contrast) of other experiences, to think of issues such as connections between militancy and experimentation, the relationships between aesthetics and artistic-political objectives, among others.

KeYwords: Theatrical activism, Bodies, Cordobazo, Aesthetics, Policy.

Repensar las relaciones entre arte y política en la historia reciente resulta sumamente productivo en la coyuntura actual, en la que se multiplica la exploración de nuevas formas de incidir en lo social-político desde el activismo artístico como respuesta a la restauración conservadora del neoliberalismo, no sólo en Argentina sino en otros países, y especialmente, en América Latina. En el contexto actual, revisar particularmente la militancia artística constituye un ejercicio fructífero no solo para observar antecedentes y contagios estéticos y modos de participación política, sino también para profundizar en las reflexiones sobre las posibilidades y las limitaciones de la incidencia artístico-política en las sociedades actuales, y para discernir objetivos y fines posibles de nuestra participación ciudadana. La conmemoración de los 50 años del Cordobazo ilumina de manera particular ese referente ineludible en la búsqueda de elementos que nos permitan crear alternativas viables para transformar nuestro entorno y proyectar nuestro futuro.

El contexto cordobés en torno a 1969 fue especialmente fértil para la emergencia de prácticas artísticas militantes, entre las cuales el teatro aparece como una de las formas más eficaces para la acción política, debido a su esencia colectiva, a su capacidad de vincular cuerpos y de construir lazos. En estas páginas es mi intención realizar un recorrido crítico por el proceso político en torno al Cordobazo a través del análisis de la experiencia del colectivo Libre Teatro Libre (LTL). Una aproximación in extenso desde esta perspectiva nos permitiría poner de relieve aspectos significativos del caso que operan como metonimia (por asociación o por contraste) de otras experiencias, y nos permiten reflexionar en torno a problemáticas como las conexiones entre militancia y experimentación; las apuestas estéticas (en la puesta en práctica de elementos de teatro documental, de teatro épico, y/o de conexión con las vanguardias experimentales, entre otras referencias estéticas del momento) en función de los objetivos artístico-políticos; y de las formas de 
asociación comunitaria a nivel local, nacional y regional. En esta ocasión concretaré una breve aproximación a algunas de estas problemáticas.

LTL surgió en el marco de la Universidad Nacional de Córdoba (UNC) movilizada por los acontecimientos sociales y políticos de 1969, y pronto se convirtió en uno de los grupos de teatro militante más reconocidos de la época. LTL funcionó como un colectivo teatral orgánico entre 1970 y 1975 , y fusionó la militancia política con la exploración estética, participó del "corredor de teatro latinoamericano" 1 de la época y puede ser pensado como "brazo teatral" del PRT (Partido Revolucionario de los Trabajadores). Es uno de los pocos colectivos originados en el interior de Argentina (es decir, en una ciudad que no sea Buenos Aires), que ha conseguido sistematicidad, continuidad y repercusión, no sólo en ese país sino en toda América Latina y en algunos países de Europa. En todo esto radica la especial atención que depositamos sobre las experiencias de este colectivo.

\section{HACIA UNA TRANSFORMACIÓN DE LAS PRÁCTICAS}

El breve lapso de tiempo que se extiende entre fines de los años ' 60 y comienzos de los ‘70 constituye un período que puede ser definido como parte de una "época” (Claudia Gilman, 2003), puesto que es posible observar una estructura de sentimiento marcada por la posibilidad de cambios radicales en los órdenes establecidos, es decir, en última instancia, por la idea de revolución, que tomó formas variadas y tuvo los más diversos alcances en diferentes latitudes de Occidente. Como sabemos, el desarrollo del pensamiento y de la acción en este período se irradió desde acontecimientos como la Revolución Cubana y el Mayo Francés, con la guerra de Vietnam y los procesos de descolonización en frica como faros que alentaban las posibilidades de transformación.

El elemento diacrítico del período está dado por la politización de todas las esferas, que se extendió a todos los aspectos de la vida cotidiana. Por consiguiente, los artistas se vieron interpelados por este clima de época en su quehacer, lo que dio como resultado una transformación en la composición de cada campo cultural. Entre las respuestas ofrecidas por los artistas al proceso de politización de la vida cotidiana, se destaca la emergencia de ciertas prácticas que adaptaron sus obras y los modos de producción a la radicalización política del contexto. Sectores provenientes de todas las disciplinas artísticas radicalizaron su actividad, estableciéndose como espacios alternativos a los circuitos legitimados. Así, las experiencias de teatro militante se desarrollaron en sintonía con otras provenientes del cine, la literatura, la música y las artes visuales, que se corresponden con la figura del intelectual-artista revolucionario emergente en este periodo. Artistas con diversas trayectorias y formaciones radicalizaron sus prácticas, de manera que es posible afirmar que en América Latina en la época de los '60-'70 surge, se desarrolla y se diluye un "teatro militante", que se concretó de diferente manera en los distintos escenarios.

En términos generales, el teatro militante de esta época puede definirse como un modo de participación militante que combinaba la acción política y la escénica, en un trabajo que involucraba a los agentes culturales en entidades colectivas y a los sectores populares como sujeto colectivo de recepción, propiciando el borramiento de los límites entre artistas y público, a través de una participación activa de la experiencia, con la finalidad de que lo vivenciado repercutiera en acciones sociales o políticas posteriores. Los colectivos, por supuesto, poseían distinto tipo de adscripción ideológica y estética, perseguían diferentes objetivos, tenían un diverso modo de producción, de circulación y de vínculo con formaciones políticas y con otros agentes del campo cultural. Algunos referentes ineludibles del teatro militante latinoamericano son el brasileño Augusto Boal, quien para esa época definía un "teatro del oprimido" y el colombiano Enrique Buenaventura, quien acuñó también en ese entonces la idea de "creación colectiva”.

Partiendo de la idea de que la noción de militancia se carga de significaciones específicas en diversas coyunturas, me interesa definir a esas prácticas culturales como "militantes" debido a que sus objetivos y cierto tipo de metodologías se asocian a aquellos cultivados por la militancia política de la época. Como hemos 
dicho, el teatro militante de la época estableció relaciones dialécticas con prácticas afines desarrolladas desde otras disciplinas (como el cine militante, la canción popular o el activismo en las artes visuales), pero también con otros sectores del campo teatral (como el realismo crítico y la experimentación vanguardista emergentes en los '60, el teatro oficial, el teatro popular-comercial o el Teatro Independiente), que tomaron otras opciones frente a las transformaciones socio-políticas. En este sentido, la fijación de nuevos patrones estéticos y políticos dio como resultado la transformación del campo cultural en general y del teatral en particular, observándose corrimientos y desviaciones con respecto a su conformación en los primeros años de la década del ' 60 .

Asimismo, tanto el teatro como la militancia son prácticas necesariamente colectivas. En ambos, la asociacioón con otros - definidos en la época como "compañeros"- aparece como un elemento distintivo y definitorio de la posibilidad de acción. En teatro, esta cualidad asociativa se articula con la reflexión con y desde el cuerpo como elemento que hace a la construcción identitaria de los sujetos y de la actividad. Además, en el teatro militante setentista, la reflexión en torno a lo local, lo nacional y lo latinoamericano emerge como parámetro fundante de los lenguajes, las prácticas y los sentidos, y obtendrá diversos modos de expresión en los diferentes colectivos. Es decir, el ser-en-común (la organización comunitaria), la acción (teatral y política), la relación con los partidos o movimientos políticos y con circuitos de difusión, y las problemáticas a nivel local (barrial, zonal, etc.), nacional y continental, tomarán una forma específica en cada caso; tales formas se originan y revierten en una toma de postura política. ${ }^{2}$

\section{LA UNIVERSIDAD y EL TEATRO EN LA CóRdoba de 1969}

El Cordobazo se estableció como epicentro de una serie de acontecimientos que convirtieron el año 1969 en el punto más alto del camino que el país venía transitando en su viraje hacia la izquierda. Si bien existe cierto acuerdo respecto de la periodización de la época, el comienzo y el fin del período son materia de discusión. Silvia Sigal y Oscar Terán han publicado en 1991 en la editorial Puntosur sendos trabajos sobre los años '60 (Intelectuales y poder en la década del sesenta y Nuestros años sesentas, respectivamente), que se constituyeron como lecturas clave. Allí, Terán sostiene que el año 1966 funcionó como un parteaguas, mientras que Sigal considera que durante los años siguientes se observa la continuidad de ciertas prácticas culturales, por lo que la fractura no se habría dado con el golpe de Estado de 1966, sino en 1969, con el Cordobazo (Sigal y Terán, 1992).

En lo que respecta al campo teatral y a la radicalización de ciertos sectores, en mi investigación tomo como inicio del período 1969, puesto que es a partir de ese momento que es posible detectar el accionar sistemático de colectivos de teatro militante. De la misma manera, el final del período estaría dado por la clausura de dichas experiencias. La dificultad para seguir haciendo ese tipo de intervenciones no ocurrió de un día para otro, sino que comenzó a mediados de 1974, con la derechización en el ejercicio del poder por parte del gobierno peronista. Dos años después, para el golpe de Estado del 24 de marzo de 1976, esas experiencias irían dejando de existir. La mayor parte de los grupos se disolvieron, y algunos pocos consiguieron transformar sus prácticas, camuflarse, adaptarse a la nueva realidad, creando tácticas para evadir la censura y evitar la persecución (Verzero, 2016).

Con el Cordobazo se materializó por primera vez en la historia argentina una versión nacional del hecho revolucionario: ese movimiento espontáneo, protagonizado por estudiantes universitarios y obreros que contaban con el apoyo de sectores de clase media, y en el que los partidos políticos y los grupos guerrilleros (que aún estaban en formación) no desarrollaron un papel de importancia, funcionó como modelo posible para el cambio de la realidad nacional en su conjunto.

Si bien este levantamiento se inserta en la marea revolucionaria que tuvo reproducciones en distintos focos del mundo, la experiencia en las sociedades dependientes posee un agregado de valor político y económico 
que pone en juego la integridad de las identidades sociales. En este sentido, Nicola\#s Casullo (1998: 185) subraya:

No somos, quizá infelizmente, los '60 franceses ni italianos ni berlineses ni californianos. No somos "el tiempo de la protesta callejera" ni el de la "revolución cultural" ni el de "las generaciones marcusianas" que hoy la industria cultural homenajea patentizando el fin de la historia en su "última historia". Córdoba fue lo más parecido a una revolución que nos pasó.

En la convulsionada Universidad Nacional de Córdoba de 1969, un grupo de estudiantes se encontraba cursando la materia "Práctica escénica I" con María Escudero a cargo de la cátedra perteneciente al Departamento de Teatro de la Escuela de Artes, y se encontró con los sucesos de mayo a poco de comenzado el año lectivo. El clima de movilización que se vivía en Córdoba marcó el habitus con el que el grupo ingresó al campo teatral y a lo político, guiado por Escudero, quien regresaba de haber vivido en Francia, donde había estudiado y trabajado en pantomima con Marcel Marceau. La propuesta de Escudero en la cátedra estaba orientada a la transformación de la escena y de la realidad, integrando la investigación formal y social en un trabajo basado en la creación colectiva y la presencia física del actor (Escudero, 1974). Se proponía trabajar con improvisación, privilegiando lo lúdico y lo colectivo.

Algunos docentes de la Escuela, entre los que se encontraba María Escudero, comenzaron a pensar en que habían sostenido un posicionamiento europeizante que los alejaba del arraigo latinoamericano. Cecilia Curtino (2017: 119) hace referencia a este posicionamiento identitario que Oscar Terán (1993) describía como "autoculpabilidad" y explica que con el Cordobazo se dio "la irrupción de lo concreto, real y problemático de la vida obrera” en la vida universitaria y:

a partir de este acontecimiento que mostró la eficacia de un colectivo en acción, el compromiso fue vivido como inevitable por algunos docentes universitarios porque se sintieron parte de un movimiento que excedía lo que ocurría en su ámbito privado o en el grupo reducido que frecuentaban en su labor diaria.

LTL estaba integrado por jóvenes de entre dieciocho y veinticuatro años con inquietudes de formación actoral que en 1969 ingresaron a la universidad. En ese sentido, el origen universitario del grupo constituye una particularidad respecto de la mayor parte de los colectivos de teatro militante. En el marco del antiintelectualismo de la época, las relaciones institucionales han constituido uno de los dilemas centrales de estos grupos, que desarrollaron distintas relaciones en distintos momentos con la institución-arte, con las salas como espacio legitimado del teatro canónico, con los partidos políticos y con la academia. Aunque prontamente la UNC los expulsó, LTL lleva impreso el perfil de sus integrantes como alumnos de la Escuela de Teatro de la universidad, que se combina con la experiencia en el cuerpo de los acontecimientos sociales de myor envergadura del país. La conjunción de la vivencia del ámbito cordobés estallado y de la exploración estética propuesta por Escudero promovió una práctica artística y política de gran compromiso social, poético e ideológico.

Lindor Bressán, integrante del grupo, en una entrevista personal, sintetizaba la significación política de la provincia en el marco nacional de la siguiente manera:

Lo que pasa es que es la vanguardia. Córdoba en ese momento era indiscutiblemente la vanguardia en montones de cosas: en la universidad, en lo sindical, en lo político: [Agustín] Tosco le cuestionaba el liderazgo político a [José Ignacio] Rucci con un sindicato de dos mil personas, frente a la CGT [Confederación General de los Trabajadores], que tenía en ese momento un sindicato con 700.000, 800.000 obreros. ¡ Un representante de dos mil obreros le cuestionaba el poder al tipo que manejaba todo! Entonces, esas son las cosas que cambiaron en ese momento históricamente. ${ }^{3}$

En julio y en diciembre de 1969 el grupo presentó tres trabajos prácticos al resto de la Escuela, que se titularon Sensaciones, La blufa de la misericordia y El ritual del hombre. Se hicieron entre dos y cuatro presentaciones de estos trabajos y se invitó a todos los estudiantes de la institución. Además, los fines de semana realizaban estos montajes para recaudar fondos con la finalidad de viajar a Buenos Aires para ver teatro. Se trataba de fiestas temáticas que incluían happenings y se hacían en el Teatrino (un espacio 
de 10 x 10 metros que había en la Ciudad Universitaria, que se utilizaba como se encontrara, con gradas o escaleras) o en el parque de la Ciudad Universitaria. El ritual del hombre se presentó en el escenario del Pabellón Argentina y en colaboración con los otros Departamentos de la Escuela. Los estudiantes de teatro también colaboraban como actores en cortometrajes que realizaban los alumnos de la carrera de Dirección de Actores, perteneciente al Departamento de Cine. La interdisciplinariedad constituía otra de las características particulares de la propuesta de Escudero.

$\mathrm{Al}$ año siguiente, los alumnos pidieron a través de notas formales a la institución que Escudero fuera la docente a cargo de la materia "Práctica escénica II" como modo de continuar y profundizar en aproximación a la actuación que habían realizado en la asignatura anterior. La solicitud fue denegada, por lo que los estudiantes hicieron protestas en la universidad y en la calle, reforzando la visibilidad que habían querido darle al asunto a través de algunas apariciones en los medios. En sintonía con la propuesta que habían experimentado con Escudero, reclamaban, además, la conformación de un taller interdisciplinario donde las materias funcionaran como un proyecto de trabajo. Estas movilizaciones tenían por finalidad que se modificara el plan de estudios, adaptándolo a las transformaciones estéticas e ideológicas que se vivían. Todo terminó cuando se le abrió un sumario a Escudero y fue apartada del cargo. El grupo abandonó con ella la universidad y continuó el trabajo de manera independiente en la casa de quien ya podía considerarse su "Maestra". Es en este marco y en 1970 que se funda formalmente LTL.

El colectivo concibió el proceso de cambio en una dialéctica entre las transformaciones socio-políticas y la gestación de nuevas formas estéticas. En ese sentido, se propuso producir un teatro político de base que recogiera las problemáticas de las clases populares y pusiera en acto lenguajes escénicos acordes a la búsqueda de transformación de los órdenes de dominación con un paralelo trabajo de exploración estética. Entre los procedimientos adoptados se encuentran la ruptura de categorías del teatro burgués, como la noción de autor o la de sala a la italiana, la puesta en primer plano del lenguaje corporal y la concepción del hecho escénico como encuentro, junto al trabajo con material documental y al proceso de creación desde la idea de "creación colectiva".

En una combinación de elementos diversos, la este\#tica de LTL está atravesada conceptualmente por las renovaciones impulsadas contemporáneamente por el Living Theater, Jerzi Grotowski, Antonin Artaud, y también por Bertold Brecht y Peter Weiss. La experimentación ligada a las vanguardias de mediados del siglo XX era absorvida por LTL a través de la triple vía del Living, Grotowski y Artaud. Esto involucraba el cuestionamiento a las formas del teatro burgués tradicional, transformando los roles que ocupaban habitualmente el autor, el director, el actor y el espectador. Entre los elementos sustanciales de estas transformaciones, es posible pensar que el lugar de autoría dramática pasa a ser colectivo; la actuación se desdobla a través de técnicas que involucran diferentes modos de percepción y que pone en primer plano un fuerte entrenamiento a partir de la conexión de cuerpos y energías; el espectador es sustraido de un lugar de recepción pasivo para pasar a ser un sujeto activo, que es interpelado, provocado o invitado a participar de la situación escénica; el espacio, por su parte, estalla en mil formas posibles de ocupación, no solo en una sala a la italiana (los espectadores frente a la situación escénica, donde los actores gozan de la seguridad de la cuarta pared imaginaria), sino que el público se ubica alrededor, a los lados o, incluso, circula a través del espacio escénico. LTL se apropia de técnicas impulsadas por Grotowski que involucran el trabajo intercorporal, distanciándose de un modo de actuación realista, central en la escena argentina e impulsada por seguidores de Stanislavsy y del método Strasberg. La propuesta del "teatro de la crueldad" de Artaud, por su parte, ofrece un sustrato filoófico a la exploración estética.

Al tiempo que LTL asumía un fuerte entrenamiento corporal ligado a estas tendencias, no desechaba perspectivas teatrales que propnen ligar la situación socio-política con la práctica artística de manera más directa. De ahí que su exploración estética vanguardista se vinculara con el teatro documental a través del cual Weiss proponía llevar acontecimientos históricos a escena a partir de ciertas pautas, y con el teatro épico de Brecht, cuyo objetivo central consistía en crear consciencia crítica en los espectadores a través de 
procedimientos que ostentaran el artificio teatral, como el distanciamiento, para evitar la identificación del público con la escena. Habitualmente, el teatro ligado a las vanguardias experimentales toma distancia de propuestas de teatro documental o épico, y a la inversa, pero LTL logra combinar eficazmente la experimentación estética con un teatro que explicita su compromiso socio-político.

La apropiación de algunos elementos se llevó a cabo a partir de la toma de contacto con materiales diversos en distintos momentos de algunos procesos artísticos y significó no sólo la aplicación de estrategias provenientes de metodologías y lenguajes renovadores, sino una transformación en la concepción del hecho escénico. Ya en su primer trabajo, La blufa de la misericordia (1969), habían roto el espacio escénico concretando el contacto con el público, y en el primer espectáculo propiamente dicho, $\mathrm{El}$ asesinato de $\mathrm{X}$ (1970), una obra basada en El asesinato de Malcom X, del autor uruguayo Iber Conteris, se incoroporó la desnudez física y espacial, en un espacio escénico circular, construyendo un ritual en el que es posible observar la intertextualidad con el Living Theater, con Grotowski y con Artaud, a quienes los integrantes del grupo conocían a través de mediaciones y cuyo teatro no habían podido ver en vivo. En la Escuela de Cine de la UNC se habían proyectado films del Living y los conocimientos sobre Grotowski y Artaud hasta ese momento llegaban a la provincia más mediados que de primera mano. Grotowski visitaría la Argentina, y más precisamente, la provincia de Córdoba en $1971 .^{4}$

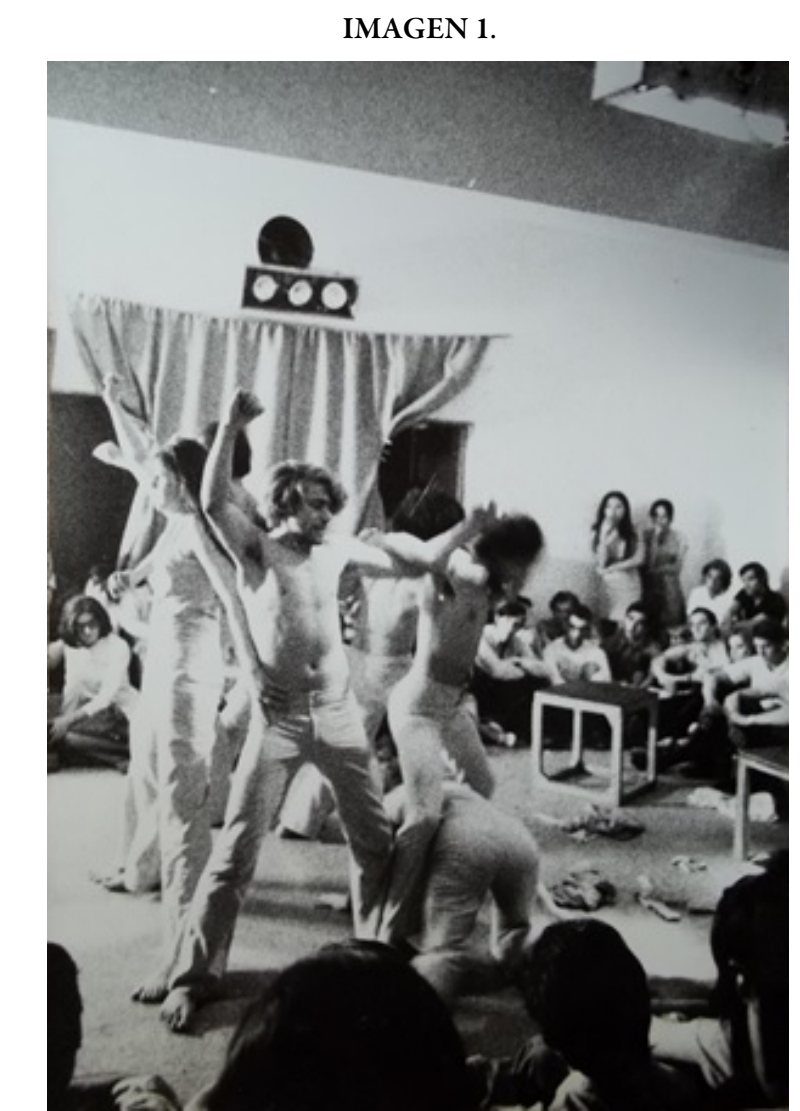

Estreno de El asesinato de X, hall del cine club Lumiere, noviembre de 1970. De izquierda a derecha: Cristina Castrillo, Lindor Bressán, Pepe Robledo; en el piso: Luisa Nuñez; detrás, de espaldas: Oscar Rodríguez, Graciela Ferrari y Paco Quinodoz. Archivo: Lindor Bressán.

La obra de Conteris, por su parte, había sido estrenada por El Galpón en Montevideo (Uruguay, 1969), luego de haber obtenido una Mención Especial en el concurso de Casa de las Américas (La Habana, Cuba) en 1968. La obra visibiliza las problemáticas de los negros como expresión de la opresión del capitalismo. El proceso de trabajo de LTL se extendió durante nueve meses en los que actualizaron la pieza al contexto cordobés, basándose en la vida de un sindicalista que, aunque no se explicita, hace referencia a Tosco. 
El asesinato de X fue galardonada con Primera Mención en el premio Casa de las Américas de La Habana, Cuba, lo que operó como impulso para la realizacioón de una gira de LTL por América Latina, que se extendió durante todo 1971. La obra fue editada por Casa de las Américas y por la revista Teatro de Medellín, Colombia, en 1972. Estos pocos datos operan como metonimia de la avidez por el intercambio de los jóvenes "trabajadores de la cultura" -como muchos de ellos se autodenominaban-, y exhiben los deseos de tender lazos y de dejar fluir saberes, técnicas y modos de hacer a través de las fronteras.

Los valores sesentistas en relación con la ruptura de las normas incuestionables de la modernidad, un reordenamiento de los vínculos sociales en torno a la libertad (moral, sexual, comunicacional) y a un nue-vo ordenamiento del mundo a partir de la posibilidad de transformación, se encontraban en el centro del horizonte de expectativas del sector de la juventud del que LTL formaba parte. Se establece en este punto una distancia ideológica con otros colectivos, como Octubre o el Centro de Cultura Nacional José Podestá ${ }^{5}$, para quienes la transformación tenía necesariamente una forma nacional basada en la impronta del peronismo como doctrina política y también social. Los integrantes de estos grupos ligados al peronismo, sin embargo, no expresan públicamente críticas a la experimentación formal de la vanguardia sesentista (incluso, entre otros, Norman Briski, referente del grupo Octubre, había realizado trascendentes experiencias en el Instituto Di Tella), pero existen posiciones más extremas, para las cuales el camino vanguardista era considerado frívolo, vacío de contenido, imperialista y extranjerizante. Este punto de vista era compartido por la tendencia realista y por sectores de la izquierda clásica más radicalizada. LTL, sin embargo, consigue articular propuestas estéticas que ponían en juego lenguajes vanguardistas (como hemos dicho, de la mano de técnicas grotowskianas, procedimientos implementados por el Living Theater y conceputalizaciones provenientes del pensamiento y de la propuesta teatral Artaud), junto a un trabajo con archivos según los planteos de Peter Weiss para la construcción de un teatro documental y herramientas de un teatro épico brechtiano, como el distanciamiento.

\section{...Y LUEGO}

En enero de 1971, la ciudad de Córdoba volvió a ser el foco de las movilizaciones obreras. Se produjo otro levantamiento popular en la provincia, conocido como el "segundo Cordobazo" o "Vivorazo". Durante gran parte del año LTL realizó una gira en la que, además de presentar El asesinato de $X$, desarrolló otras experien-cias en Chile, Bolivia, Ecuador y Colombia. En este último destino, El asesinato de $X$ formó parte de la cuarta edición del Festival Latinoamericano de Manizales, que se desarrolló en septiembre de 1971, donde encontraron un reconocimiento inesperado. En estos meses fueron reescribiendo la pieza, complejizándola de acuerdo a las realidades que iban transitando, concretando la idea de una obra abierta.

$\mathrm{Al}$ regreso de la gira latinoamericana, LTL estrenó Contratanto (1972), una obra de teatro documental producida junto con la Unión de Educadores de la provincia de Córdoba que gira en torno a los temas del sistema educativo y del rol del docente como transmisor de ideología. Esta pieza obtuvo el premio Trinidad Guevara de los Servicios de Radio y Televisión de la Universidad, en reconocimiento a la investigación llevada a cabo, y fue publicada por la revista española Primer Acto en 1973. Además, Contratanto fue seleccionada para representar a la Argentina en el V Festival de Manizales (1973), donde tuvo muy buena repercusión periodística y un gran éxito de público. 


\section{IMAGEN 2}

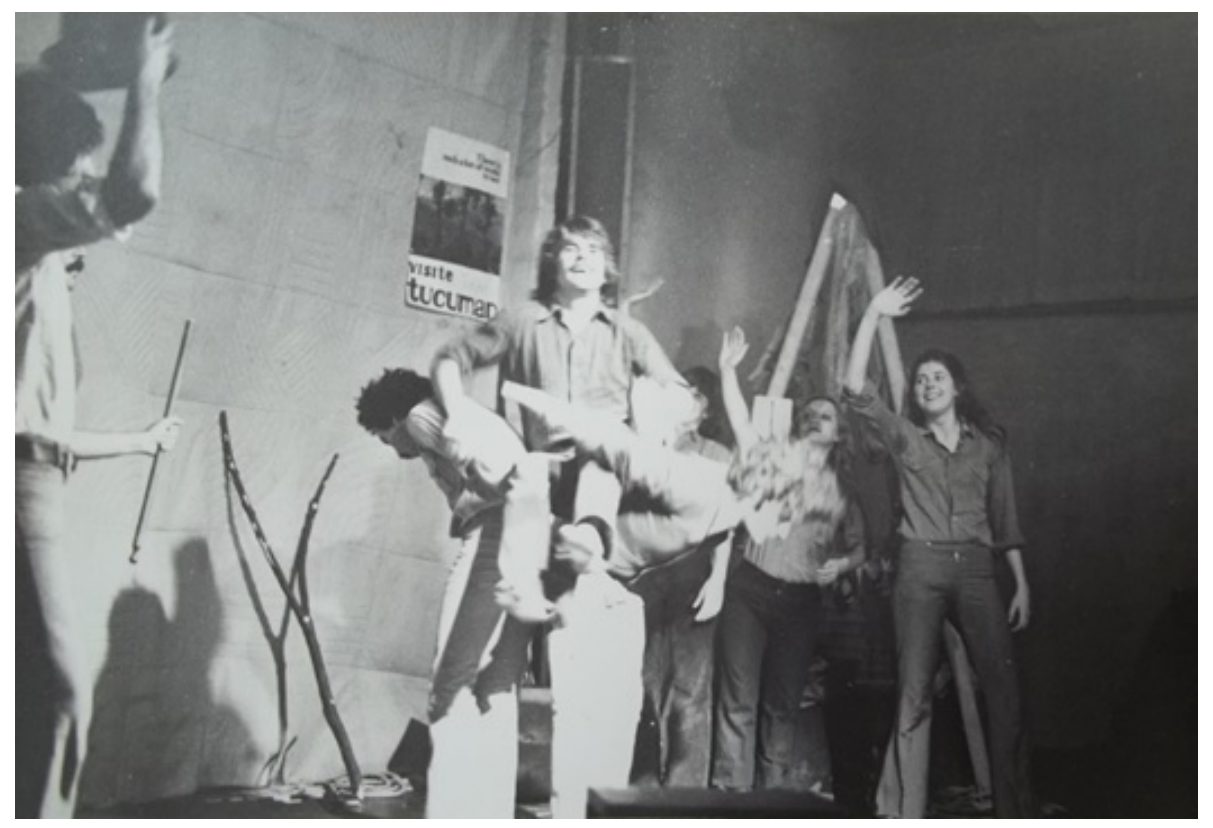

El fin del camino, Teatrino de la Ciudad Universitaria de la UNC, 1974. Oscar Rodríguez, Pepe Robledo, Lindor Bressán, Julio Saldaña, Luisa Nuñez, Graciela Ferrari, Cristina Castrillo. Archivo: Lindor Bressán.

Entre septiembre y mediados de diciembre de 1973, se asentaron en Tucumán, donde realizaron una investigación sobre la realidad de los ingenios azucareros. Como resultado de ese trabajo de campo, luego de un prolongado proceso que se extendió entre marzo y octubre de 1974, estrenaron El fin del camino. Esta obra recibió el premio Trinidad Guevara por parte de la Universidad Nacional de Córdoba.

"El fin del camino", la frase que da título a la que sería la última obra del grupo, es la traducción al español de tucma, término quechua del que proviene el nombre de la provincia. En una especie de continuidad con Tucumán arde (la experiencia de contrainformación generada por artistas visuales en 1968 que resultó luego paradigmática), y con El camino hacia la muerte del "Viejo" Reales (una película del mismo año, realizada por Gerardo Vallejo, afín al colectivo cinematográfico Cine Liberación), LTL propone una pieza que denuncia la situación social en un entrecruzamiento entre documentación y estetización, es decir, entre el trabajo con materiales de archivo y la creación escénica.

El viaje a Tucumán y el trabajo sobre los ingenios azucareros partió de una propuesta del PRT, el partido en el que para ese entonces militaba la mayor parte de los integrantes del colectivo como parte de un frente cultural. Podría decirse, incluso, que LTL funcionaba como "brazo teatral" del partido, es decir, constituia el nexto teatral entre el partido y la sociedad, ponía al alcance de sectores sociales más amplios sus lineamientos ideológicos a través de propuestas escénicas. La noción de "brazo artístico" ha sido subrayada respecto del cine militante: Cine de la Base (con Raymundo Gleyzer a la cabeza) es frecuentemente definido como "brazo cinematográfico" del PRT y Cine Liberación (con la coordinación de Fernando "Pino" Solanas y Octavio Gettino), del peronismo de izquierda. En el mismo sentido, algunos colectivos teatrales -entre los que se destaca LTL- operaban como vasos comunicantes entre sus agrupaciones políticas de referencia y sectores sociales no necesariamente militantes.

Las relaciones entre LTL y miembros del PRT comenzaron a gestarse en 1972, cuando el colectivo teatral participó de festivales nacionales o locales en defensa de los presos políticos.

La militancia política de LTL puede organizarse en dos etapas: una primera, entre 1972 y 1974, en la que el grupo realizó actividades culturales y políticas en diferentes lugares marginales con adhesión explícita al PRT; entre ellos, el trabajo con sindicatos o la organización del FAS (Frente Antiimperialista y por el Socialismo), un frente impulsado por el PRT donde convergían diferentes tendencias. Y una segunda etapa, que consiste en su trabajo como parte del Frente de Trabajadores de la Cultura. Los documentos oficiales del 
PRT reconocen la existencia de FATRAC (Frente de Trabajadores de la Cultura) entre 1968 y 1971, cuando se decretó la disolución del brazo cultural del partido para dar paso a la centralidad de las acciones de guerrilla. ${ }^{6}$ Sin embargo, como vemos, es posible observar una continuidad en las prácticas culturales gestadas desde trabajadores de la cultura del partido. La colaboración de LTL en este Frente cultural se dio, entonces, en un narco de participación cultural no sistematizada pero reconocida al interior del partido, que se continuó luego de la disolución "oficial” del FATRAC. En ese sentido, Bressán explica:

Nosotros, el LTL, éramos del FAS [Frente Antiimperialista por el Socialismo]. Nos presentábamos como militantes del FAS, trabajábamos en la conformación del Frente, donde el Frente de Trabajadores de la Cultura era parte integrante del FAS. Nunca decíamos públicamente que pertenecíamos al PRT. Fuimos a los Congresos del FAS que se hicieron en el Chaco y en Rosario, en noviembre del ' 73 en Resistencia y en julio del ` 74 en Rosario (fechas aproximadas). En Tucumán, nos invita gente del Comité Central del PRT, pero vamos a trabajar con él en nombre del FAS. ${ }^{7}$

Las relaciones con el partido fueron tensas, sobre todo los momentos en que se impulsaba la participación en la lucha armada. Los tiempos de la práctica cultural son habitualmente más lentos que otros modos de acción y, a lo largo de mi investigación, he recogido testimonios de trabajadores de la cultura que participaron de grupos con distinta alineación política, como trostkistas, peronistas o comunistas, que coinciden en manifestar las discrepancias con los compañeros de militancia que no veían en la práctica artística una capacidad transformadora, al menos, en los plazos cortos que ellos buscaban. Entre los dilemas centrales de la relación entre el PRT y LTL se encuentra la urgencia de la militancia y el trabajo a largo plazo de la práctica artística para la conscientización social (que se profundizó durante la llamada “desviación militarista”); el hecho de que los compañeros militantes consideraban que el teatro era una actividad menor, recreativa, que los distanciaba de las tareas que debían realizar como miembros del partido; la imposibilidad de realización de tareas de militancia por parte de los integrantes de LTL que supusieran algún nivel de clandestinidad ser figuras reconocidas -sobre todo en la ciudad de Córdoba-, y el consiguiente dificultoso pase a la clandestinidad de estos individuos conocidos por su trabajo como actores (debido al cual no sólo eran vistos por el público que asistía a sus obras sino también, por ejemplo, por los lectores de los diarios que sacaban notas sobre su trabajo teatral acompañadas de fotografías). ${ }^{8}$

Hacia 1971--1972, la consigna de politización del arte de vanguardia ya resultaba antigua. Ese objetivo había sido reemplazado por el de dotar a la política de estatuto artístico. No se trataba ya -como en los últimos ' 60 - de experiencias estéticas con connotaciones políticas, sino de experiencias políticas estetizadas y, en no más de dos o tres años, se transformarían en experiencias políticas o armadas sin visos de estetización alguna.

En febrero de 1974 Córdoba volvió a hervir: se produjo el "Navarrazo", un golpe de Estado al gobierno provincial electo en los comicios de septiembre de 1973. El gobernador y el vicegobernador, Ricardo Obregón Cano (ligado a sectores de la izquierda peronista) y Atilio López (secretario general la UTA, Unión Tranviaria Automotor) fueron destituidos y asumió el poder el jefe de la policía de la provincia, Antonio Navarro. Mientras tanto, LTL realizaba algunos espectáculos y se sumergía en el proceso de elaboración de los materiales que habían relevado en Tucumán, para estrenar en octubre de ese año El fin del camino, en el Teatrino de la UNC. La obra tuvo once funciones y debió ser levantada debido a la densidad de los conflictos sociales y al riesgo evidente que suponía la temática planteada.

En medio de amenazas, en 1975 el grupo LTL decidió salir del país. Se trasladaron a Venezuela, donde llevaron cinco obras con las que habían trabajado en los años anteriores (dos espectáculos infantiles: Glup, zas, pum, crash! O la verdadera historia de Tarzán y La mayonesa se bate en retirada; Contratanto; Algo por el estilo y El fin del camino). 


\section{IMAGEN 3}

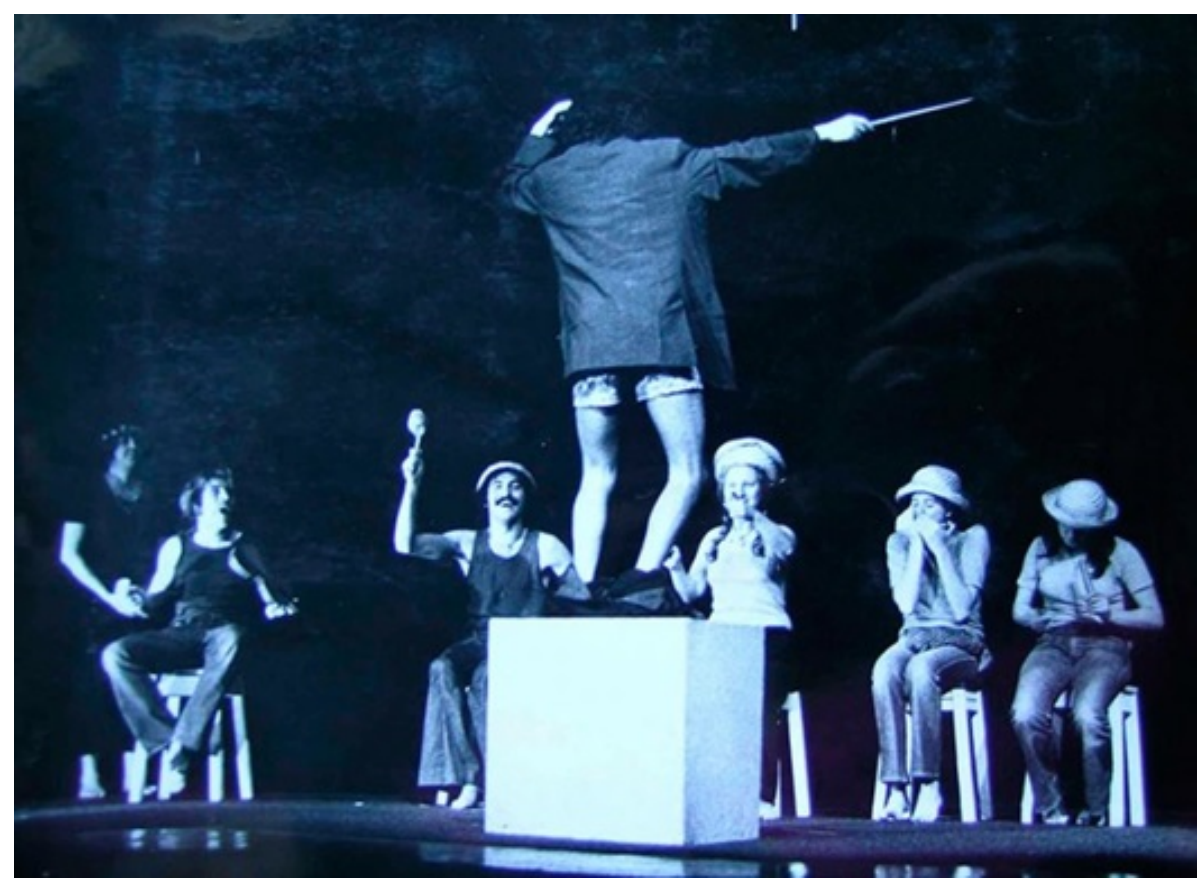

La mayonesa se bate en retirada, 1975. Fuente: Carolina Lucero, “Un nuevo teatro: Libre Teatro Libre”, La izquierda diario, 29 de mayo de 2016: https://www.laizquierdadiario.com/Un-nuevo-teatro-Libre-Teatro-Libre

Hicieron una gira por todo Venezuela, donde encontraron el reconocimiento de la intelectualidad latinoamericana, y tomaron contacto con Daniel Viglietti, Soledad Bravo y Juan Bosch, entre otros artistas. Comenzaban a encontrarse trabajadores de la cultura que desde algunos países ya estaban saliendo al exilio. Allí, entre los debates sobre si volver a la Argentina o continuar en el exilio, el grupo se disolvió. Pepe Robledo, Roberto Videla y Graciela Ferrari se quedaron en Venezuela y, más tarde, se dirigieron a Italia. Videla actualmente reside en la ciudad de Córdoba. El resto del grupo regresó a la Argentina, para salir no mucho tiempo después a un exilio prolongado. Lindor Bressán viajó a México, un año después pasó a España, y años más tarde regresó a la Argentina. Oscar Rodríguez y Susana Rueda salieron hacia Brasil y luego se fueron a España, donde aún residen. Bressán y Rodríguez se reencontraron en España un año despueés de haberse exiliado. Susana Pautazzo salió hacia a Brasil, para radicarse, luego, en Ecuador. Cristina Castrillo se exilió en Suiza. Luisa Núñez es la única integrante del grupo que se quedó en Córdoba. María Escudero falleció en Ecuador en 2005. Los nombres son más que nombres, exhiben trayectorias, caminos, recorridos, lazos tendidos que transportan saberes, cajas de experiencias que se contagian, se nutren unas de otras, se enriquecen y se transforman en cada desplazamiento.

\section{Conclusiones}

Subyace a estas consideraciones la idea de que el arte no reproduce ni repite lo social, y tampoco se ve determinado por el contexto socio-político, sino que constituye una fuerza activa que es parte de un proceso histórico. En ese sentido, acontecimientos como el que eclosionó en Córdoba en mayo de 1969 se definen necesariamente como epicentro de formas de producción artística y cultural en relación dialéctica con otros modos de asociación.

La centralidad de la política en la época aparece en el campo cultural de diversas maneras, entre las que se destacan los corrimientos, las negociaciones y las transformaciones, entre las que detectamos la emergencia de un teatro militante. La funcionalidad social de las prácticas teatrales militantes se inscribe, entonces, en el ideal revolucionario y, de acuerdo con la adscripción ideológica, política o partidaria de cada uno de 
los grupos, las experiencias teatrales militantes se materializaron en diversos modos de compromiso con lo social--político y diferentes maneras de concebir la propia práctica (en términos políticos y estéticos).

El momento climático que representó vivir la ciudad de Córdoba hacia 1969 fue caldo de cultivo para la configuración de nuevas prácticas, de nuevos modos de asociación, entre los que el teatro no puede estar ausente.

Mientras que algunos colectivos de teatro militante con diversa alineación política y partidaria pusieron el acento en la participación política, otros reivindicaron la experimentación estética. Entre ellos, LTL concibió el proceso de cambio en una dialéctica entre las transformaciones socio-políticas y la gestación de nuevos lenguajes artísticos. Esta especial caracterización de LTL a caballo entre la militancia política y la exploración estética, con un compromiso comprobable en ambas esferas, tiene asiento en una confluencia de factores complejos, entre los cuales, sin dudas, resalta la vivencia en el cuerpo de los acontecimientos del Cordobazo como experiencia fundacional.

\section{REFERENCIAS}

Bressán, Lindor (2014). "Cuando la provincia fue metrópolis". Seminario "Antropología de las culturas nacionales: Intercambios mundializados y reinvención de tradiciones nacionales”, Departamento de Antropología, Facultad de Filosofía y Humanidades, Universidad Nacional de Córdoba, inédito.

Casullo, Nicolás (1998). Modernidad y cultura crítica. Buenos Aires: Paidós.

Curtino, Cecilia (2017). "Un escenario moderno. Los textos institucionales del Departamento de Teatro, cánones de lectura”. En Musitano, Adriana (dir.) Teatro, politica y universidad. El Departamento de Teatro, un escenario moderno. Córdoba/Buenos Aires: Facultad de Filosofía y Humanides-UNC y Facultad de Filosofía y LetrasUBA.

Escudero, María (1974). “María Escudero: la historia del grupo Libre Teatro Libre o la radiografía de una utopía que se convirtió en la gran opción actual del teatro cordobés”, La Voz del Interior, Coórdoba, 5 de mayo: 9-10 .

Gilman, Claudia (2003). Entre la pluma y el fusil. Debates y dilemas del escritor revolucionario en América Latina. Buenos Aires: Siglo Veintiuno.

Longoni, Ana (2005). “El FATRAC, frente cultural del PRT/ERP”. Lucha Armada, año 1, No 4, septiembre-octubre-noviembre: 20-33.

Sigal, Silvia (1991). Intelectuales y poder en la década del 60. Buenos Aires: Puntosur.

Sigal, Silvia y Terán, Oscar (1992) “Los intelectuales frente a la política”. Punto de Vista, año xv, No 42, abril: $42-49$.

Silva Mariños, Lisandro (2017). Frente antiimperialista y por el socialismo. Un ejército politico de masas impulsado por el PRT. Buenos Aires: La llamarada.

Terán, Oscar (1993 [1991]). Nuestros años sesenta. La formación de la nueva izquierda intelectual en Argentina (1956-1966). Buenos Aires: El Cielo Por Asalto.

Verzero, Lorena (2013). Teatro militante. Radicalización artística y politica en los años '70. Buenos Aires: Biblos.

Verzero, Lorena (2016). "Entre la clandestinidad y la ostentación: Estrategias del activismo teatral bajo dictadura en Argentina”. En Remedi, Gustavo (ed.).El teatro fuera de los teatros. Reflexiones criticas desdeel archipiélago teatral. Montevideo: Universidad de la República: 87-104.

Verzero, Lorena (2019) "Redes regionales y transatlánticas de teatro militante latinoamericano: Una cartografía en construcción”, en Transcultur@. Diccionario de historia cultural transatlántica. Siglos XVIII-XXI. París/ São Paulo: Agencia Nacional de Investigación (ANR) (Francia) y Fundación de Apoyo a la Investigación del Estado de Sao Paulo (FAPESP) (Brasil). Recuperado de https://tracs.hypotheses.org/2094 


\section{Notas}

1 En Verzero, 2013: 369-380, propongo la hipótesis de la existencia de "un corredor de teatro militante latinoamericano" y realizo un itinerario crítico que la confirma.

2 Para ver en profundidad sobre estas problemáticas, ver: Verzero, 2013.

3 Esta entrevista con Lindor Bressán se realizó en Buenos Aires el 10 de octubre de 2007.

4 En Verzero, 2019, me extiendo sobre la visita de Grotowski y sus implicancias estéticas y simbólicas.

5 He realizado una construcción crítica del trabajo de estos dos colectivos en: Verzero, 2013. Se trata de dos colectivos militantes ligados a agrupaciones peronistas de izquierda, con diverso tipo de organización, de metodología de trabajo y de conformación de los grupos.

6 Ana Longoni (2005) ha realizado una aproximación a este tema.

7 Correo electrónico de Lindor Bressán a Lorena Verzero, 5 de abril de 2019. El V Congreso del FAS al que se refiere Bressán fue exactamente en Chaco en diciembre de 1973 y el VI Congreso, en Rosario, en junio de 1974. Sobre este Frente, ver: Lisandro Silva Mariños, 2017.

8 Sobre las relaciones de LTL con el PRT, he realizado una aproximación en: Verzero, 2013: 229-239. 